\title{
Challenges in fatty acid and lipid physiology
}

\author{
Jan F. C. Glatz* \\ Department of Molecular Genetics, Cardiovascular Research Institute, Maastricht University, Maastricht, Netherlands \\ ${ }^{*}$ Correspondence: glatz@maastrichtuniversity.nl
}

Lipids comprise a wide variety of substances that share the common feature of being insoluble in water but soluble in organic solvents. Fatty acids are important building blocks of lipids and they give a diversity and chemical specificity to the complex lipids found in natural fats and oils, comparable to that given by the amino acids to proteins. Because of their special characteristics and significance, fatty acids are commonly treated as a separate group. Together, fatty acids and lipids are increasingly being recognized as serving important roles in physiology. Thus, fatty acids and lipids serve as membrane constituents and in energy supply and fuel storage, but specific lipids also function in regulating a wide variety of cellular processes, including gene expression. The most notable example is that long-chain fatty acids and some oxygenated derivatives can bind and thereby activate nuclear receptor proteins, in particular the so-called peroxisome proliferator activated receptors (PPARs), which in turn influence the expression of specific genes like those involved in lipid metabolism. In this way, fatty acids act as signaling molecules regulating the cellular routes and capacity for lipid utilization. Within this context, also the role of retinoic acid, a derivative of vitamin $\mathrm{A}$, as a major regulator of gene expression should be mentioned. Another development is the finding that the level of lipid storage, for instance in adipose tissue, can be sensed by specific compounds. The corollary is that lipid droplets can no longer be viewed as inert intracellular deposits but rather appear dynamic structures able to communicate with other intracellular compartments. Taken together, a dynamic interplay among lipids, proteins, and membranes is of central importance for the proper physiological regulation of cellular functions. Below, I will highlight examples of new developments in this exciting field together with challenges for future research.

\section{BIOLOGICAL MEMBRANES}

The function and properties of membranes are to a great extent determined by the chemical structure of their lipid components, their orientation in the membrane and interaction with other membrane constituents, in particular membrane-associated proteins. The discovery of specific membrane lipid domains such as lipid rafts has added another level of complexity and regulation of the functioning of membranes and membrane-related processes, in particular signaling events. Lipid rafts are fluctuating nanoscale assemblies of sphingolipid, cholesterol, and proteins that can be stabilized to coalesce, forming platforms that function in membrane signaling and trafficking (Lingwood and Simons, 2010). For instance, in the secretory pathway, sorting of not only proteins but also lipids has to occur before exit from the trans-Golgi network. Selective lateral segregation of membrane proteins and lipids is thought to be a key event in promoting membrane bending and subsequent budding to form transport vesicles. Similarly, caveolae, plasma membrane invaginations that form a subdomain of lipid rafts, function in endocytosis, transcytosis, and regulation of various signaling events (Lajoie and Nabi, 2010). Interestingly, $\omega-3$ fatty acids have been shown to alter caveolae composition and thereby modify endothelial function (Layne et al., 2011), indicating that caveolae represent a regulatory platform for nutritional modulation.

\section{FATTY ACID AND LIPID TRANSPORT}

The hydrophobic nature of (long-chain) fatty acids and lipids dictates specific requirements to their transport in aqueous solutions. In blood plasma, fatty acids are avidly bound by albumin while lipids are transported in lipoproteins such as chylomicrons and very-low density lipoprotein (VLDL). With respect to the mechanism of cellular uptake of fatty acids, it has been shown that the lipid bilayer of the plasma membrane does not represent a barrier for fatty acids (Hamilton, 2007). However, several membrane-associated fatty acidbinding proteins (FABPs) have been identified and shown to markedly accelerate cellular fatty acid uptake. These membrane proteins (often referred to as "fatty acid transporters") function not only in facilitating but also in regulating fatty acid entry into the cell. This is currently viewed to occur by adsorbing fatty acids from the extracellular media, modulating their transport into the membrane, and segregating or organizing fatty acids for metabolism (Glatz et al., 2010). In addition, one of these fatty acid transporters, i.e., CD36, was found to regulate fatty acid uptake in muscle by a mechanism that resembles that of GLUT4mediated cellular glucose uptake. Thus, following an acute stimulus (insulin, muscle contraction) CD36 translocates from an intracellular store (endosomes) to the plasma membrane. This recycling of CD36, together with the similar recycling of the glucose transporter GLUT4, appear to be pivotal for the proper regulation of cellular substrate uptake, for instance to clear lipids from the circulation postprandially and to rapidly facilitate substrate provision when the metabolic demands of muscle are increased by contractile activity (Glatz et al., 2010). Recent data indicate that this function of CD36 is controlled not only by intracellular recycling but also by posttranslational modification, in particular $\mathrm{N}$-glycosylation (Lauzier et al., 2011).

Intracellular transport of fatty acids and lipids is facilitated by specific proteins, such as the abundantly expressed (cytoplasmic) FABPs (Storch and Corsico, 2008). Members of this latter group of proteins appear to be involved in trafficking their ligands within the cytosol via interactions with organelle membranes and specific intracellular proteins. In addition, some members of the FABP family of proteins have been shown to function directly in the regulation of cognate nuclear transcription factor activity via ligand-dependent translocation to the nucleus and, therefore, may modulate, for instance, PPAR-regulation of gene expression. Taken together, a number of proteins are involved in the transport of fatty acids and lipids throughout the body, and in this way determine their availability and functioning. Such controlled availability is important because fatty acids, largely due 
to their hydrophobic properties, also exert harmful effects and may cause (acute) cellular injury.

\section{LIPID SENSING AND LIPID SENSORS}

Exciting new insights have been obtained in the field of lipid sensing. Besnard and colleagues disclosed that the membrane protein CD36 functions as an oral sensor for long-chain fatty acids (Passily-Degrace et al., 2009). Taste cells express CD36 which interacts with unsaturated fatty acids, resulting in enhanced pancreatobiliary excretions, indicating interorgan signaling to prepare for the digestion of increased dietary fat. In addition, as mentioned above, there now is clear evidence that the body is capable of sensing the amount of lipid stored in adipocytes (Dugail and Hajduch, 2007).

\section{FATTY ACIDS AND LIPIDS IN SIGNALING PATHWAYS}

In the last decades a host of new knowledge has become available on signal transduction pathways regulating cellular functioning. Among the molecules that participate in those signal chains lipids and lipid derivatives constitute a major portion. For instance, phosphatidylinositol and related compounds are involved in a number of important intracellular events, e.g., in insulin signaling. With respect to cell-cell communication, the term lipid mediators has been introduced to denote a class of bioactive lipids that are produced locally through biosynthetic pathways in response to extracellular stimuli, are then sequestered to transmit signals to target cells by interacting with specific receptors. Lipid mediators thus can be regarded as (local) hormones (Murakami, 2011). The various lipid mediators comprise arachidonic acidderived eicosanoids, including prostaglandins, leukotrienes, and related compounds, lysophospholipids and derivatives such as platelet-activating factor and also including endocannabinoids, and finally the newly identified anti-inflammatory lipid mediators derived from $\omega-3$ polyunsaturated fatty acids, i.e., resolvins (derived from eicosapentaenoic fatty acid, EPA) and protectins (derived from docosahexaenoic fatty acid, DHA; Ariel and Serhan, 2007). It is clear that lipid mediators not only play an essential role in sustaining homeostasis, but also function in pathogenic mechanisms including the inflammatory response.

\section{FATTY ACIDS AND LIPIDS IN DISEASE: THE METABOLIC SYNDROME}

In several chronic diseases such as cardiovascular and neurodegenerative diseases with genetic and lifestyle components (e.g., dietary factors), there is also a perturbed lipid metabolism. An example that currently receives world-wide attention is the so-called metabolic syndrome, defined as a clustering of multiple metabolic abnormalities based on central obesity and insulinresistance. The significance of the metabolic syndrome is its close association with the risk of type 2 diabetes and cardiovascular disease. The current obesity epidemic has led to a strong increase in the prevalence of type 2 diabetes and, in turn, in its cardiovascular complications. Thus, obesity is characterized by disturbances in the control of glucose and fat metabolism which is associated with an atherogenic lipid profile in blood and with deleterious fat accumulation in non-adipose tissues like liver, heart, and muscle (ectopic fat deposition). As a consequence, individuals suffering from these abnormalities are at high risk for developing cardiovascular complications, including stroke, myocardial infarction, and kidney failure (Kassi et al., 2011). Further understanding the regulation of wholebody lipid metabolism, its perturbations in obesity and insulin-resistance, and the cause of this cascade of adverse events eventually leading to cardiovascular disease, is expected to help design strategies to prevent (e.g., lifestyle intervention) and treat this chronic metabolic disorder. For instance, one of the issues currently heavily debated is whether $\omega-3$ polyunsaturated fatty acids (EPA, DHA) have beneficial health effects or that caution should be taken with their recommendation, because of possible adverse (e.g., pro-arrhythmic) effects (Saravanan et al., 2010).

\section{LIPIDOMICS}

Over the past decade, the "omics" evolution has expanded to embrace lipidomics as a major contributor to our understanding of biological processes in health and disease (Meikle and Christopher, 2011). Lipidomics refers to the identification and quantification of the many distinct molecular species, using state-of-the-art approaches made possible by newly developed instrumentation, protocols, and bioinformatics tools. As such, lipidomics is expected to provide a powerful approach to understanding lipid biology (Brown and Murphy, 2009). In a recent study, more than 500 lipids representing the major species of the sphingolipid, glycerolipid, glycerophospholipid, fatty acyl, sterol, and prenol classes present in human plasma were quantified by a "targeted lipidomic" strategy to provide a base for future studies to characterize their relationship to human physiology, nutrition, and disease (Quehenberger et al., 2010). Also in the above-mentioned field of the metabolic syndrome, lipidomics has now begun to be applied to gain new insight (Meikle and Christopher, 2011).

\section{CONCLUSION}

Among the various biological molecules that comprise the human body, i.e., nucleic acids, amino acids, carbohydrates, and lipids, lipids stand out in the sheer number of distinct molecular species. Lipids serve essential roles in many aspects of human functioning. In fact, it has become increasingly difficult to find (patho)physiological processes in which lipids do not play important, if not central, roles as signaling and regulatory molecules. The challenge of this field is to gain further insight into the dynamic interplay both among the lipid molecular species and among lipids, proteins, and biological membranes. The latter includes not only lipids interacting with proteins, but also lipid-modification of proteins, e.g., post-translational $N$-myristoylation (Martin et al., 2011), which adds another level of functionality to many proteins. The still expanding availability of research models, such as cellular models and transgenic rodents, and of novel analytical methodologies, such as high-resolution mass spectroscopy, will dramatically accelerate the generation of new data. Subsequently it will become crucial to discuss these data properly and to disclose the physiological significance of the observations. As an example, human plasma has been reported to contain thousands of distinct lipid molecular species distributed among the main lipid categories (Quehenberger et al., 2010). Given the powerful analytical tools currently available and the expected further improvement of these techniques in the near future, it will be a major challenge to discern the roles of individual lipids in health and disease before future blood tests will include 
their routine assessment. The intention of Frontiers in Fatty Acid and Lipid Physiology is to provide a forum for in-depth discussion of such new research findings in this exciting area.

\section{REFERENCES}

Ariel, A., and Serhan, C. N. (2007). Resolvins and protectins in the termination program of acute inflammation. Trends Immunol. 28, 176-183.

Brown, H. A., and Murphy, R. C. (2009). Working towards an exegesis for lipids in biology. Nat. Chem. Biol. 5 , 602-606.

Dugail, I., and Hajduch, E. (2007). A new look at adipocyte lipid droplets: towards a role in the sensing of triacylglycerol stores? Cell. Mol. Life Sci. 64, 2452-2458.

Glatz, J. F. C., Luiken, J. J. F. P., and Bonen, A. (2010). Membrane fatty acid transporters as regulators of lipid metabolism: implications for metabolic disease. Physiol. Rev. 90, 367-417.

Hamilton, J.A. (2007). New insights into the roles of proteins and lipids in membrane transport of fatty acids. Prostaglandins Leukot. Essent. Fatty Acids 77, 355-361.

Kassi, E., Pervanidou, P., Kaltsas, G., and Chrousos, G. (2011). Metabolic syndrome: definitions and controversies. BMCMed. 9, 48. doi: 10.1186/1741-7015-9-48
Lajoie, P., and Nabi, I. R. (2010). Lipid rafts, caveolae, and their endocytosis. Int. Rev. Cell Mol. Biol.282, 135-163. Lauzier, B., Merlen, C., Vaillant, F., McDuff, J., Bouchard, B., Beguin, P. C., Dolinsky, V. W., Foisy, S., Villeneuve, L. R., Labarthe, F., Dyck, J. R. B., Allen, B. G., Charron, G., and Des Rosiers, C. (2011). Post-translational modifications, a key process in CD36 function: lessons from the spontaneously hypertensive rat heart. J. Mol. Cell. Cardiol. 51, 99-108.

Layne,J., Majkova,Z.,Smart,E.J., Toborek,M., and Hennig, B. (2011).Caveolae: a regulatory platform for nutritional modulation of inflammatory diseases. J. Nutr. Biochem. doi:10.1016/j.jnutbio.2010.09.013. [Epub ahead of print].

Lingwood, D., and Simons, K. (2010). Lipid rafts as a membrane-organizing principle. Science 327, 46-50.

Martin, D. D. O., Beauchamp, E., and Berthiaume, L. G. (2011). Post-translational myristoylation: fat matters in cellular life and death. Biochimie 93, 18-31.

Meikle, P. J., and Christopher, M. J. (2011). Lipidomics is providing new insight into the metabolic syndrome and its sequelae. Curr. Opin. Lipidol. 22, 210-215.

Murakami, M. (2011). Lipid mediators in life sciences. Exp. Anim. 60, 7-20.

Passily-Degrace, P., Gillard, D., and Besnard, P. (2009). Orosensory perception of dietary lipids in mammals. Results Probl. Cell Differ. 1, 1-18.
Quehenberger, O., Armando,A.M., Brown, A. H., Milne, S. B., Myers, D. S., Merrill, A. H., Bandyopadhyay, S., Jones, K. N., Kelly, S., Shaner, R. L., Sullards, C. M., Wang, E., Murphy, R. C., Barkley, R. M., Leiker, T. J., Raetz, C. R. H., Guan, Z., Laird, G. M., Six, D. A., Russell, D. W., McDonald,J.G., Subramaniam, S., Fahy, E., and Dennis, E. A. (2010). Lipidomics revelas a remarkable diversity of lipids in human plasma. J. Lipid Res. 51, 3299-3305.

Saravanan, P., Davidson, N.C., Schmidt, E. B., and Calder, P.C. (2010). Cardiovascular effects of marine omega-3 fatty acids. Lancet $376,540-550$.

Storch, J., and Corsico, B. (2008). The emerging functions and mechanisms of mammalian fatty acid-binding proteins. Annu. Rev. Nutr. 28, 73-95.

Received: 21 July 2011; accepted: 21 July 2011; published online: 02 August 2011.

Citation: Glatz JFC (2011) Challenges in fatty acid and lipid physiology. Front. Physio. 2:45. doi: 10.3389/ fphys.2011.00045

This article was submitted to Frontiers in Fatty Acid and Lipid Physiology, a specialty of Frontiers in Physiology.

Copyright $\odot 2011$ Glatz. This is an open-access article subject to a non-exclusive license between the authors and Frontiers Media SA, which permits use, distribution and reproduction in other forums, provided the original authors and source are credited and other Frontiers conditions are complied with. 